\title{
Prediction of Performance Penalty due to Pump-Signal Overlap in Raman-amplified Systems
}

\author{
Gabriele Di Rosa $^{1,2^{*}}$, Md Asif Iqbal ${ }^{3}$, André Richter ${ }^{1}$ and Wladek Forysiak ${ }^{3}$ \\ ${ }^{1}$ VPIphotonics GmbH, Carnotstr. 6, Berlin, Germany \\ ${ }^{2}$ Technische Universität Berlin, Straße des 17. Juni 135, Berlin, Germany \\ ${ }^{3}$ Aston Institute of Photonics Technologies, Aston University, Birmingham B4 7ET, UK \\ *gabriele.di.rosa@vpiphotonics.com
}

\begin{abstract}
We present an efficient numerical model to predict the performance penalty induced by Rayleigh backscattered light arising from counter-propagating pumps in Ramanamplified ultra-wide-band transmission systems. The model is validated through comparison with experimental findings. (C) 2020 The Author(s)
\end{abstract}

\section{Introduction}

The ever-increasing need for higher data throughput, together with the requirement to find cost-effective solutions, is fostering research in the development of ultra-wide-band systems operating on the available fiber infrastructure [1]. Wavelength division multiplexed (WDM) transmission over C- and L-bands is approaching the spectral efficiency limit while bandwidth extension offers a more rewarding increase of the throughput [2]. One of the main challenges related to multi-band systems is the development of suitable amplification schemes. Recently, $\mathrm{S}+\mathrm{C}+\mathrm{L}$ band flat amplification has been demonstrated with different techniques, employing rare-earth doped fiber amplifiers, semiconductor optical amplifiers and Raman amplification [2-4]. It has been shown in particular how multi-stage backward pumped discrete Raman amplifiers (DRAs) are able to provide improved noise figure over a large bandwidth [4]. When the WDM signal bandwidth exceeds the peak Raman gain shift of $\approx 100 \mathrm{~nm}$, wavelength regions in which both signals and pumps are present arise. Evident transmission penalties induced by Rayleigh backscattered light (RBS) have been experimentally observed in this scenario [5].

We develop an efficient numerical model to easily predict the transmission penalty induced by RBS generated by counter-propagating pumps in Raman amplification schemes. The model is validated by comparison with the experimental results obtained in [5] and provides a very good agreement while keeping low the algorithmic complexity.

\section{Simulation setup and model derivation}

The simulation setup is shown in Fig. 1. The input signal ranges from 1485 to $1625 \mathrm{~nm}$ and is propagated over 70 $\mathrm{km}$ of standard single mode fiber (SSMF) before entering the dual stage backward pumped DRA under analysis. In S-band we consider two 30 GBaud quadrature phase-shift keying modulated signals shaped using a raised cosine filter with 0.2 roll-off factor. Their central wavelengths are swept respectively around $1485 \mathrm{~nm}$ and $1508 \mathrm{~nm}$ in a range of $\pm 3.2 \mathrm{~nm}$ to span over the overlapping broadband pumps spectra, whose peaks are found around these same two central wavelengths. In C- and L-band the channels are represented simply as single spectral lines to take into account the Raman power transfer in an efficient way. The DRA is composed of two inverse dispersion fiber (IDF) spans of length $L=7.5 \mathrm{~km}$. This fiber is characterized by a linear Rayleigh backscattering coefficient of $\gamma_{r}=1.6 \times 10^{-7} \mathrm{~m}^{-1}$. In order to establish a common reference performance level between the experiment in [5] and our model, we introduce additive white Gaussian noise (AWGN) at the input of the system. The noise power is set to obtain a reference $\mathrm{Q}^{2}$ factor $\approx 20 \mathrm{~dB}$ at $1508 \mathrm{~nm}$ and $\approx 17.2 \mathrm{~dB}$ at $1485 \mathrm{~nm}$ at the output of the amplifier when RBS is not taken into account.
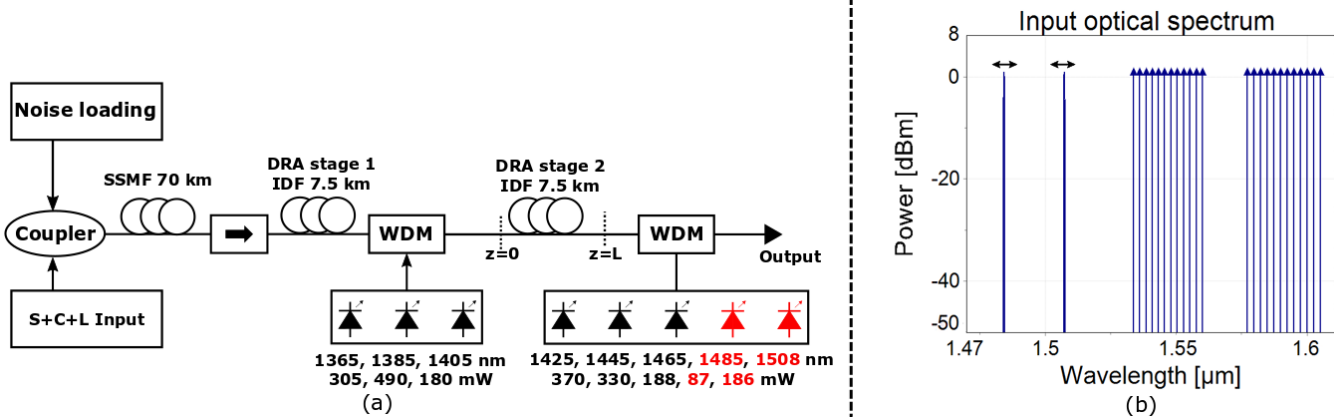

Fig. 1: (a) Simulation setup. In red the pumps that overlap with the signal. The wavelengths and respective powers of the pumps are reported. The DRA provides an average gain $\approx 15 \mathrm{~dB}$. (b) Optical spectrum of the $\mathrm{S}+\mathrm{C}+\mathrm{L}$ input. 
Recalling that the $\mathrm{Q}$ factor has for noise-limited systems an equivalent description in the signal to noise ratio (SNR), we can now define $\operatorname{SNR}_{r e f}\left(\lambda_{i}\right)$ as the value that corresponds to our reference $\mathrm{Q}^{2}$ factor for $\lambda_{i}=\{1485,1508\} \mathrm{nm}$. To evaluate then the RBS induced penalty we calculate the total SNR as in (1).

$$
\operatorname{SNR}_{t o t}(\lambda)=\frac{P_{s}^{+}(z=L, \lambda)}{P_{s}^{+}(z=L, \lambda) / \operatorname{SNR}_{r e f}\left(\lambda_{i}\right)+P_{R B S}^{+}(z=L, \lambda)}
$$

Where $P_{S}^{+}(z=L, \lambda)$ and $P_{R B S}^{+}(z=L, \lambda)$ are respectively the signal and RBS forward propagating power at the output of the DRA. In order to estimate these two quantities, it is needed to describe the longitudinal evolution of $P_{s}^{+}$and of the counter-propagating Raman pump power, which we refer to as $P_{p}^{-}\left(z, \lambda_{i}\right)$. Extensive literature regarding the numerical solution of this problem is available. During our work we evaluated it through the model included in VPIphotonics Design Suite 10.0. For this operation the broadband pumps are described as single spectral components located at wavelengths $\lambda_{i}$. This approximation proved to provide reliable results while significantly decreasing the algorithmic complexity. Once $P_{s}^{+}$and $P_{p}^{-}$have been obtained on the domain $z \in[0, L]$ the problem reduces to the estimation of $P_{R B S}^{+}(z=L, \lambda)$. In our scenario, where the pump power is much higher than the overlapping signal, it is reasonable to neglect double RBS arising from the signal. Hence, we can estimate $P_{R B S}^{+}(z=L, \lambda)$ as the sum of all the RBS contributions from the pump generated along the DRA, appropriately scaled by the gain or attenuation they are subject to when propagating from the generation point to $z=L$. If we spatially divide our amplifier into infinitesimally small sections $d z$ we can then write (2).

$$
P_{R B S}^{+}(z=L, \lambda)=\int_{0}^{L} \gamma_{r} P_{p}^{-}(z, \lambda) \frac{P_{s}^{+}(L, \lambda)}{P_{s}^{+}(z, \lambda)} d z
$$

Here $P_{p}^{-}$is the pump power that falls inside the receiver bandwidth. This term takes into account the spectral shape of the broadband pump, which proved to be a key parameter in order to obtain reliable predictions.

\section{Model validation}

From the derived $\mathrm{SNR}_{t o t}$ we calculate the $Q^{2}$ factor and verify our model through comparison with the experimental penalty reported in [5]. As can be observed in Fig. 2, the model provides a very good estimation of the performance penalty versus the wavelength separation between the central wavelengths of the overlapping pump and signal. The agreement is good at $1485 \mathrm{~nm}$, where a worst case $\mathrm{Q}^{2} \mathrm{drop} \approx 2 \mathrm{~dB}$ is present, and at $1508 \mathrm{~nm}$, where higher pump power and Raman gain cause a much larger RBS induced penalty $\approx 15 \mathrm{~dB}$. The different shape of the two graphs is due to the different pumps' spectra [5], which are then taken into account in our model.

$\mathrm{Q}^{2}$ around $1485 \mathrm{~nm}$

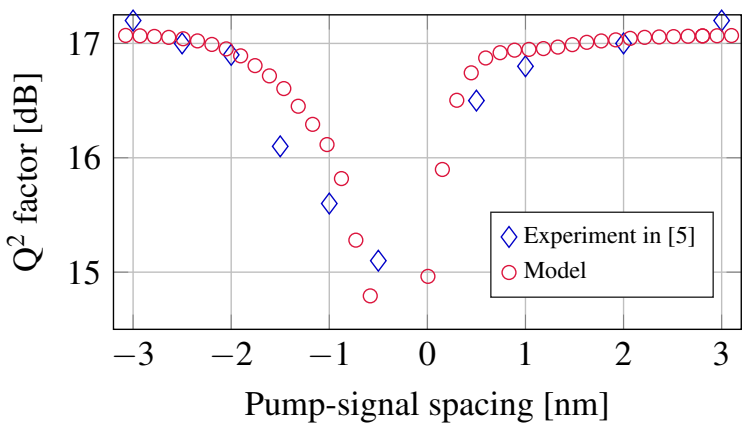

$\mathrm{Q}^{2}$ around $1508 \mathrm{~nm}$

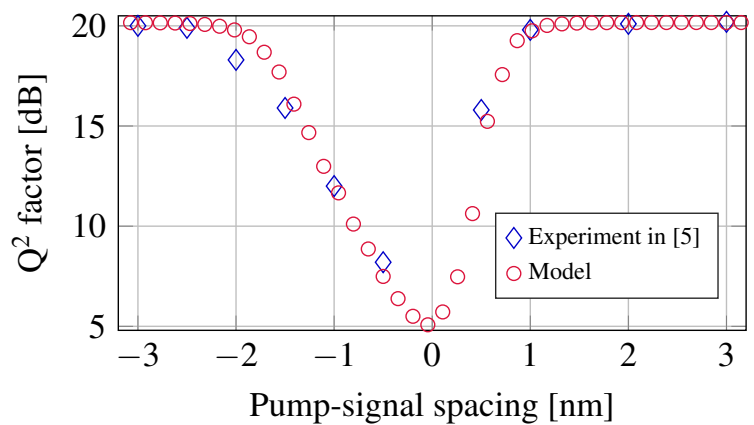

Fig. 2: Comparison between the simulation results and the experimental penalty observed in [5].

\section{Conclusions}

We presented an efficient method to predict the performance penalty arising from pump-signal overlap in Ramanamplified ultra-wide-band systems. We demonstrated excellent agreement of our model predictions with experimental results, both proving its reliability and corroborating the findings in [5]. In particular we confirmed that the penalty observed is entirely due to RBS and is strongly influenced by the spectral shape of the pump.

\section{Acknowledgments}

This work is funded by the EU under H2020-MSCA-ITN-2018 Grant Agreement 814276 (WON) and the German Ministry of Education and Research under the grant 16KIS0993 (OptiCON)

\section{References}

1. A. Napoli et al., "Towards multiband optical systems," Photonic Networks and Devices, OSA, 2018.

2. J. Renaudier et al., "First 100-nm continuous-band WDM transmission system with $115 \mathrm{~Tb} / \mathrm{s}$ transport over $100 \mathrm{~km}$ using novel ultrawideband semiconductor optical amplifiers," Proc. ECOC, Th.PDP.A.3 (2017).

3. F. Hamaoka et al., "Ultra-wideband WDM transmission in S-, C-, and L-bands using signal power optimization scheme," J. Lightw. Technol. 37(8), pp. 1764-1771 (2019).

4. M. A. Iqbal et al., "Noise performance improvement of broadband discrete Raman amplifiers using dual stage distributed pumping architecture," J. Lightw. Technol. 37(14), pp. 3665 - 3671 (2019).

5. M. A. Iqbal et al., "Evaluation of performance penalty from pump-signal overlap in S+C+L band discrete Raman amplifiers," Proc. OFC, W4B.2 (2020, to be presented) 